\title{
Forecasting and Visualization of Wildfires in a 3D Geographical Information System
}

\author{
M. Castrillón ${ }^{\mathrm{a}}$, P.A. Jorge ${ }^{\mathrm{b}}$, I.J. López ${ }^{\mathrm{c}}$, A. Macías ${ }^{\mathrm{b}}$, D. Martín ${ }^{\mathrm{b}}$, R.J. \\ Nebot $^{c}$, I. Sabbagh ${ }^{\text {, F.M. Quintana }}{ }^{\text {, J. Jánchez }}{ }^{\mathrm{b}}$, A.J. Sánchez ${ }^{\mathrm{b}}$, J.P. \\ Suárez $^{\mathrm{d}}$, A. Trujillo ${ }^{\mathrm{b}}$ \\ ${ }^{a}$ SIANI, University of Las Palmas de Gran Canaria, Spain \\ ${ }^{b}$ Computer Science Department, University of Las Palmas de Gran Canaria, Spain \\ ${ }^{c}$ Software Engineering Department, Canary Islands Institute of Technology, Spain \\ ${ }^{d}$ Department of Cartography and Graphic Engineering, University of Las Palmas de \\ Gran Canaria, Spain
}

\begin{abstract}
This paper describes a wildfire forecasting application based on a 3D virtual environment and a fire simulation engine. A novel open source framework is presented for the development of 3D graphics applications over large geographic areas, offering high performance 3D visualization and powerful interaction tools for the Geographic Information Systems (GIS) community. The application includes a remote module that allows simultaneous connection of several users for monitoring a real wildfire event. The system is able to manage realistic composition of what is really happening in the area of the wildfire with dynamic $3 \mathrm{D}$ objects and location of human and material resources in real time, providing a new perspective to analyze the wildfire in-
\end{abstract}

Email addresses: mcastrillon@siani.es (M. Castrillón), ijlopez@itccanarias.org (I.J. López), rneboth@itccanarias.org (R.J. Nebot), isabbagh@itccanarias.org (I. Sabbagh), fquintana@itccanarias.org (F.M. Quintana), jsanchez@dis.ulpgc.es (J. Sánchez), jsuarez@dcegi.ulpgc.es (J.P. Suárez), atrujillo@dis.ulpgc.es (A. Trujillo) 
formation. The user is enabled to simulate and visualize a wildfire spreading on the terrain integrating spatial information on topography and vegetation types with weather and wind data. The application communicates with a remote web service that is in charge of the simulation task. The user may specify several parameters through a friendly interface before the application sends the information to the remote server responsible of carrying out the wildfire forecasting using the FARSITE simulation model. During the process, the server connects to different external resources to obtain up-todate meteorological data. The client application implements a realistic 3D visualization of the fire evolution on the landscape. A Level Of Detail (LOD) strategy contributes to improve the performance of the visualization system. Key words: Wildfire Forecasting, 3D Visualization, FARSITE, GIS

\section{Introduction}

During the last years, million hectares of forests have been destroyed worldwide by fire. Only in Spain, approximately 75000 hectares were burnt 4 during the first seven months of 2009 . Wildfire comes with catastrophic consequences, especially for natural sites. In this sense, forest fire engineers need new tools for the decision making process, not only when a wildfire occurs in a real environment, but also in a preventive manner.

We present in this paper a virtual wildfire forecasting system that has been implemented attending to the interest of different Canary Islands local authorities to protect sensitive natural areas after some recent episodes of wildfires. The particular orography and nature richness of these volcanic islands present challenging difficulties in planning and managing emergencies 
that have been so far tackled using basically paper maps at different locations communicated via telephone lines. The final objective of the system is to provide a realistic $3 \mathrm{D}$ visualization of the whole area of interest, that should serve as an assistant to local services for wildfire situation analysis and management when the catastrophe occurs, including the live visualization of the different emergency units deployed on the terrain.

The requirements collected during the analysis of the system imposed that the forecasting system should not only visualize the wildfire over a realistic 3D landscape, but also estimate its evolution according to vegetation characteristics of the geographical area and weather conditions. For fire modeling, the system makes use of FARSITE (Finney, 1998), which is similar to the system described in Hoang et al. (2008). FARSITE is a popular fire behavior and growth simulator, developed by the Department of Agriculture of the United States. FARSITE uses spatial information on topography and fuels, along with weather and wind data. It deals with different kinds of fuel models depending on the vegetation that exists on the area. This allows getting realistic $3 \mathrm{D}$ simulations that will help local authorities, not only to prevent emergencies, but also to coordinate the task force in emergency situations.

The 3D virtual environment is based on a novel framework called Capaware $\rrbracket^{1}$ which has also been developed by the work team involved in the project presented in this paper. It is a cross-platform open source software that has been developed in $\mathrm{C}++$ using the graphics toolkit Open Scene Graph

\footnotetext{
${ }^{1}$ http://www.capaware.org
} 
$(\mathrm{OSG})^{2}$ and the wxWidget: $\mathrm{S}^{3}$ library. With Capaware anyone may instantly create a visual environment with many layers of terrain information, offering a plug-in capability to allow software developers to enhance its functionalities or create new ones.

The interface includes a time panel that helps forest engineers to visualize and contrast past and ongoing fire propagation over the terrain. This provides an easy to use graphic tool for a better understanding of fire simulations. During the fire expansion, burned areas are displayed by a dark overlay to make the visualization more realistic.Additionally, a color scale can be used to distinguish the burned areas.

The system is currently being evaluated by the emergency services of the Canary Islands Regional Government. After this evaluation, the new version could include new tools to the decision making process both during real situations and for preventive measures design.

Wildfire prediction and visualization has been a topic of interest for more than ten years, mainly thanks to recent advances in information technology (Ahrens et al., 1997; McCormick and Ahrens, 1998). As a result, 3D wildfire visualization has become an object of study, not only for its technical aspects (Hoang et al., 2008; Sherman et al., 2007b; Su et al., 2006), but also the wide range of possibilities that a $3 \mathrm{D}$ scenario can show during a wildfire. 3D Visualization provides realistic depiction that is useful for forest engineers and fire officials. For that purpose, the very first approaches already pointed out the dependency upon winds, temperatures and moisture of fire behavior,

\footnotetext{
${ }^{2}$ http://www.openscenegraph.org

${ }^{3}$ http://www.wxwidgets.org
} 
being considered still in recent solutions like in Reinhardt and Crookston (2003).

Commercial solutions applied to wildfire visualization in graphic environments are common, for example the 3D Nature packages GeoConnexion, 2008) and Visual Nature Studio (VNS) software analyzed in Williams et al. (2008). In addition open source tools have been studied in immersive scenarios (Sherman et al., 2007b; Su et al., 2006), for example VRFire (Sherman et al., 2007a) and VFire (Hoang et al., 2008) packages.

3D fire visualization has also shown its interest in other applications such as in training for emergency situations. In this sense, virtual reality or visualization tools can be used in training situations where it would be dangerous or expensive to participate in a real scenario (Hoang et al., 2008; Smith and Ericson, 2009; Su et al., 2006). In Hoang et al. (2008) the immersive application represents terrain, vegetation and fire. Other applications in hazardous situations are described in Basic et al. (2003).

Wildfire visualization requires a model to simulate fire behavior. A review of fire modeling can be found in Sullivan (2008), but different approaches are present in recent literature (Douglas et al., 2006a b; Mandel et al., 2005; Serón et al., 2005).

The paper is organized as follows: Section 2 gives a brief description of the 3D geographical system. Section 3 describes the implementation of the forecasting system using a wildfire simulation engine. Section 4 deals with the wildfire representation and visualization inside the system. And finally, some remarks and future works are presented in the conclusions. 


\section{The 3D Geographical framework}

Virtual managers are powerful tools in critical situations where lots of data are present and the response time is critical. For that reason, several applications have been developed to wildfire extinguishing, management and simulation using web technologies. Multimedia and Virtual Reality have also been applied more recently in Sherman et al. (2007a); Thon et al. (2007), with a clear intention to assist technicians in wildfire management.

\subsection{The 3D Virtual Environment}

The graphical interface is provided by the Capaware framework,a 3D Multilayer Geographical Environment. Figure 1 shows the application interface with one of the Canary Islands - Gran Canaria - and several geographical layers over it. Capaware has the usual GIS software features, allowing the integration of geographical layers and 3D objects over the virtual terrain. An additional feature of the software offers the visualization of dynamic objects over the 3D generated world, providing a new perspective to analyze the information. In addition, Capaware gives the user the opportunity to manage the resources and objects placed in the terrain.

Figure 1: Geographical layers. Example of the Capaware application showing several geographical layers over Gran Canaria Island.

Different resources, like humans or vehicles, are represented as 3D objects in the scenario. Additionally, if these resources have attached GPS devices, the framework provides tools to see their real-time position and track data, 
giving technicians a kind of movie shot of what is happening in the area. Each GPS device sends periodically updated information about its position to a server. This server keeps files with the track data of every device. For those devices that are active in the system, Capaware requests information to the server every four seconds, and updates visually their position on the terrain. Also, users could request track data of a device in a specific time interval.

The management of a wildfire involves such a large number of institutions, human and material resources, that makes the coordination of all those elements and factors particularly complex without a powerful supporting system. In the area of decision making, it is unfeasible to provide an effective command if the available information is biased or contradictory.

The features of the 3D system Capaware allows to obtain a realistic composition of what is really happening in the area of the wildfire, as seen in Fig. 2. The users can have a graphical representation of metadata attributes of all the elements involved in the operation in such a quick and easy to understand way. Finally, users can access the enormous amount of available internet data layers by means of the Web Map Service (WMS) that belongs to the Open Geospatial Consortium (OGC) $)^{4}$ standards.

\subsection{Framework architecture}

The Capaware architecture is composed of three different levels. The first level comprises the operating system and the basic graphical libraries such as the already mentioned Open Scene Graph and wxWidgets libraries.

\footnotetext{
${ }^{4}$ http://www.opengeospatial.org/
} 
The second level is the Capaware core, containing basic components that permit the development of 3D applications with many layers of geographical information. The third level contains utility libraries and the plug-in interface that allows developing new functionalities. The wildfire simulator was created using this interface, being thus an external plug-in of Capaware.

The Capaware framework includes a peer to peer connection among users that provides interesting communication strategies in a real wildfire situation. For example, scene elements during a simulation as firewalls or 3D virtual models can be gained by remote users, allowing status modifications for those elements based on the true situation of the wildfire. The LayerTree interface panel offers access to the whole set of scene entities. From the LayerTree panel, a user may select which entities will be shared with other users. The communication with a remote user is established using an IP address. After a successful peer to peer connection, a copy of the shared entity is transferred to the remote machine. Thanks to a sender-receiver protocol implemented with sockets, connected users may modify the shared entities guaranteeing coherence. Then, other users could share these entities, allowing several users to share a group of entities in the 3D scenario. To prevent access to restricted entities in a 3D scene, every station of the system network publishes a tree of entities that may be accessible by any remote user.

Figure 2: Management tasks. Example of the Capaware application in management tasks. 


\section{The Wildfire Simulation Engine}

The implementation of the forecasting system has been carried out by a plug-in software that communicates with Capaware.

\subsection{Using FARSITE as a wildfire simulation engine}

This plug-in makes use of a Web Service that encapsulates open-source Core FARSITE as a kernel-based wildfire simulator. As mentioned in the introduction, FARSITE is a fire behavior and growth simulator that uses topography, the distribution of heterogeneous combustion material, weather and wind data, making it a valid solution in different environments.

In the particular context of the application described in this paper, the service schema employed ensures the interoperability with any other new visualization tool. More in detail, the advantages of using a service architecture are:

- Interoperability: Both the service and the data returned can be reached with standard technologies.

- Simplicity: Although the final user has the option to overwrite the data, the server has a copy of all static data required by the simulation engine. This avoids technical staff from wasting time using data customization in FARSITE.

- Up-to-date information: A weather server managed by the Canary Islands Institute of Technology is responsible to provide an up-to-date information of the meteorology affecting a wildfire simulation. 
- Reliability: This project team has carried out extensive benchmarks with FARSITE, trying to catch out scenarios and input data which drives a simulation to a wrong final result. This knowledge has been transferred to the service to analyze all the requests, in order to identify the problems before the simulation execution.

- Functionality: In order to provide an effective and useful service, new features were added to the open-source module Core FARSITE. These new functionalities can be very useful for future developers, such as the option to dynamically add, modify and remove new ignition points and firewalls in the land.

This schema abstracts technicians of complex details and provides new functionalities. Figure 3 shows an overview of this schema.

Figure 3: Wildfire simulation global schema with Capaware.

\subsection{The simulation cycle of life}

Once the process started, the plug-in asks the user for all the data needed for the simulation execution. Most of the information is related to the weather, the terrain extension, the simulation time periods, and the location of fire ignition points and firewalls. Other essential information, such as vegetation fuel model, moisture and the topography of the terrain, are provided by the server automatically. The vegetation combustion model is collected as a tabulated grid of values representing different combustion materials. The next step is to adapt the input data to the format required 
by FARSITE, and send it to its core. A Meteorological Forecasting System located in Gran Canaria island based on MM5 and satellite images provides to the FARSITE core the weather forecast for the selected region during the next 48 hours. This prediction is used by the core as the last required input. After that, the service starts the simulation. The whole process is monitored by the service itself which, once it detects that the simulation is over, collects all the output data and compiles it into a matrix dataset. Finally, the web service serves the output dataset through a standard OPeNDAP server and returns to Capaware the URL where the file can be obtained, as seen in Fig. 3 .

On the top of the features described above, a module has been developed to download the results of simulations (NetCDF files) and split it into highlevel $\mathrm{C}++$ classes for developing issues.

The output file encapsulates a set of matrices that stores, at each instant of time, the perimeter positions of the wildfire besides a large set of metadata describing, among other parameters, the fire spread rate, flame height, fire intensity and time arrival of the fire front. All this information is used by Capaware as an internal dynamic object that can be represented in 3D, see Fig. 4 .

Figure 4: Wildfire perimeters from the simulation process. 


\section{Wildfire Visualization}

The visual representation of wildfires in a virtual environment is a complex task, since those wildfires may affect a large patch of land, and the amount of information to manage is usually huge. After a simulation, FARSITE provides information about the fire perimeters, the intensity of flames in each perimeter, the time of arrival of the fire to a point, the velocity of the front, etc. In order to visualize the propagation of a blaze in a realistic manner, we have to cope with high demands of computing resources.

\subsection{Graphic representation}

The fire visualization is based on two particle systems to model separately the flame and the smoke. The particle system is a standard technique presented in Reeves (1983), and it is used to simulate fuzzy phenomena in a graphic environment. Particle systems are controlled by an emitter, which is the source of the particles, and it defines their location in the 3D space. The emitter manages different parameters to control the behavior of the particles, like the number of particles per unit of time, the initial velocity, their lifetime and color, or their predominant direction. The appearance of the particles may vary in order to simulate several physical phenomena like, for instance, the presence of wind, different burning materials, or the time evolution of the flames.

The graphics toolkit provides several mechanisms to efficiently model the fire perimeters through the use of placer objects. Every placer comprises a set of particle systems that are arranged linearly. It allows us to represent the perimeters as flame polygons with different intensities and life times. 
Figure 5: Wildfire visualization. Visualization showing the color palette used to represent the time stamp of the different burned areas. Red color represents the recently burned areas, and blue color represents the areas burned a long time ago.

The burned area is drawn with a darker texture during the animation process. It grows at the same time as the fire front evolves. It is also possible to draw the burned area using different colors for the perimeters. This allows the user to analyze its origin and the time evolution like in Fig. 5.

\subsection{Blaze animation}

The propagation of the blaze is carried out by means of a curve morphing technique. There exists a broad number of different morphing techniques (see Gomes et al. (1999) for a survey). Normally, in the case of wildfires, the set of perimeters are concentric curves that progressively extend on its normal direction. This allows us to compute a direct morphing transformation.

New interpolated perimeters are generated between the ones obtained by the simulation engine. Given an initial perimeter, new points are dynamically introduced for the combinatorial compatibility with the following curve, as seen in Figure 6. This compatibility is necessary to ensure that the source and target curves have the same number of vertices. They are equally distributed in the polygon to avoid an inappropriate concentration. Then, there is a match between source and target vertices with minimum distance and preserving the topological disposal. Finally, the animation is carried out by linearly interpolating these matching pairs.

Each interpolated curve is projected onto the 3D terrain and new vertices 
are introduced for a better adaptation to the orography of the land. The number of interpolated perimeters depends on the time step chosen by the user and the level of detail strategy that is explained in the following section.

Figure 6: Combinatorial compatibility. During the morphing process, new vertices are inserted in the source curve, $\mathbf{P}_{i}$, to make the number of points equal to the target curve, $\mathbf{P}_{i+1}$.

\subsection{Level Of Detail strategy}

In order to reduce the amount of information, the fire visualization approach is modified attending to the distance from the user to the terrain. A Level Of Detail (LOD) strategy is implemented to reduce the underlying mesh vertices, the number of particles and the number of flames per perimeter, depending on the distance from the camera.

The purpose of the LOD strategy is to keep up the appearance of the scene as realistic as possible, while reducing the graphic complexity, see Lindstrom et al. (1996); Suárez and Plaza (2009) for two examples in terrains, and L.D. Floriani (1996); Heok and Daman (2004); Pajarola and Gobbetti (2007) for surveys of methods. When the user is far from the wildfire, it is not necessary to show much detail, but when the scene is closer, the graphic details are increased.

The chosen LOD strategy represents the terrain as a quadtree data structure which is pre-computed and stored in disk at the phase of terrain setup. The top-most node in the quadtree depicts the terrain at the lowest level 
of detail using tiled blocks of Triangulated Irregular Networks (TIN) models, L.D. Floriani (1996). The subsequent four descendants represent the terrain at a higher level of detail, and so on to the bottom of the quadtree. When the user navigates on the terrain, the system visualizes the most favorable mesh representation, in terms of the distance terrain-observer. A disk load and clean memory algorithm performs the management of the quadtree in real time.

Figure 7: Level Of Detail for the particle systems. The size of the particle systems are modified depending on the distance to the camera.

Fire perimeters are also adapted during the navigation. This is accomplished by modifying the behavior of the particle systems and the resolution of the perimeters. Changing the parameters of the emitter, we may modify the number and the velocity of the particles, as seen in Figure 7. New vertices are inserted or removed from the curves depending on the distance. The closer the user is to the fire, the bigger the amount of vertices of the curves. The vertices are selectively inserted to preserve the shape of the polygons.

The graphics toolkit applies the LOD process to increase the resolution of the mesh and textures of the landscape. We start the adaptation of the perimeters at the same time that the graphics toolkit initiates the landscape LOD to ensure a better fit to the orography of the land. The shape of the fire should look similar independently of the camera viewpoint, the distance to the blaze, and the varying precision of the underlying mesh.

Finally, the performance of the graphic visualization is enhanced by chan- 
ging the the animation time step. The number of interpolated curves are augmented or decreased depending on the system overload and the distance from the viewpoint to the scene.

\section{Conclusions}

In this paper we have presented a novel system for the management of emergencies related to wildfires. The wildfire forecasting application is developed within the Capaware framework, which is an open source cross-platform framework to develop 3D geographical multilayer applications. This framework allows the visualization of very large 3D landscapes with an easy to use graphical user interface. With its plug-in system capability, the inclusion of the wildfire forecasting tool has been straightforward. The software has the usual GIS features, and it allows not only the integration of geographical layers over the 3D land, but also the insertion of 3D designed objects. An additional feature of the software allows the visualization of dynamics 3D objects in the terrain, providing a realistic and efficient way to view and analyze the information.

The wildfire system makes use of the FARSITE simulation engine to offer predictive functions to the forest engineers. We have designed a remote web service that is invoked from the client application. This service receives some information from the client, and connects to different servers to obtain meteorological information. It simulates the behavior of the fire with the help of FARSITE, sending the results back to the Capaware client.

A more realistic visualization of the fire progression allows us to analyze its behaviour and to consider/take some preventive measures in advance. 
The visualization of the blaze is carried out by means of a morphing process, using two different particle systems for the flames and the smoke. With the 3D graphics capabilities of Capaware and the wildfire forecasting application, the forest engineers and managers have a powerful tool in the decision making process.

A forthcoming version of the system may include new functionalities such as a greater interactivity with the FARSITE simulation engine. The users will be able to change the simulation parameters in real time, and analyze the subsequent effects. New tools to study the fire behavior like its velocity, the predicted burned area, the propagation time or the time of arrival to populated areas, would allow the users to deploy efficient preventive measures. Multiple parallel simulations would provide a broad overview of the possible situations with different meteorological conditions.

Our 3D wildfire system has been recently used in the wildfire simulation and depiction of a fire occurred in La Palma Island (Spain), where 2700 hectares were burnt. We expect an increasing use of the tool with the aim to help reducing the devastating consequences of wildfires.

\section{Acknowledgments}

This work has been partially supported by the Canary Islands Institute of Technology (ITC, Canary Islands Government, Spain), the Spanish Ministry of Education and Science and FEDER through the research projects TIN 2007-66766 and MTM2008-05866-C03-02/MTM. We also thank to the colleagues of Cabildo de La Palma (Forest Department) for their cooperation in this project. 


\section{References}

Ahrens, J., McCormick, P., Bossert, J., Reisner, J., Winterkamp, J., 1997. Case study: wildfire visualization. In: Visualization '97. pp. 451-454.

Basic, F., Cartwright, W., Handmer, J., August 2003. Geographic visualization tools for communicating the risk of floods. In: Proceedings for the 21st International Cartographic Conference.

Douglas, C. C., Beezley, J. D., Coen, J., Li, D., Li, W., Mandel, A. K., Mandel, J., Qin, G., Vodacek, A., 2006a. Demonstrating the validity of a wildfire dddas. In: Computational Science ICCS 2006. pp. 522-529.

Douglas, C. C., Lodder, R. A., Ewing, R. E., Efendiev, Y., Qin, G., Coen, J., Kritz, M., Beezley, J. D., Mandel, J., Iskandarani, M., Vodacek, A., Haase, G., 2006b. Dddas approaches to wildland fire modeling and contaminant tracking. In: Proceedings of the 38th conference on Winter simulation. pp. $2117-2124$.

Finney, M. A., 1998. FARSITE: Fire area simulator - model development and evaluation. Tech. Rep. RMRS-RP-4.

GeoConnexion, 2008. 3d nature creates wildfire visualization. http:// portal.acm.org/citation. cfm?id=359080.359083.

Gomes, J., Darsa, L., Costa, B., Velho, L., 1999. Warping and Morphing of Graphical Objects. Morgan Kaufmann.

Heok, T., Daman, D., 2004. A review on level of detail. In: Proceedings of 
the International Conference on Computer Graphics, Imaging and Visualization (CGIV04).

Hoang, R. V., Mahsman, J. D., Brown, D. T., Penick, M., Jr., F. C. H., Brown:, T. J., March 2008. Vfire: Virtual fire in realistic environments. In: Proceedings of IEEE Virtual Reality Conference 2008.

L.D. Floriani, P. Marzano, E. P., 1996. Multiresolution models for topographic surface description. The Visual Computer 12(7), 317 - 345.

Lindstrom, P., Koller, D., Ribarsky, W., Hodeges, L., Faust, N., Turner, G., 1996. Real-time, continuous level of detail rendering of height fields. In: ACM Computer Graphics, Conf. Proc. Annual Conference Series, SIGGRAPH96.

Mandel, J., Bennethum, L. S., Chen, M., Coen, J. L., Douglas, C. C., Franca, L. P., Johns, C. J., Kim, M., Knyazev, A. V., Kremens, R., Kulkarni, V., Qin, G., Vodacek, A., Wu, J., Zhao, W., Zornes, A., 2005. Towards a dynamic data driven application system for wildfire simulation. In: nternational Conference on Computational Science. pp. 632-639.

McCormick, P. S., Ahrens, J. P., March 1998. Visualization of wildfire simulations. IEEE Computer Graphics and Applications 18 (2), 17-19.

Pajarola, R., Gobbetti, E., 2007. Survey of semi-regular multiresolution models for interactive terrain rendering. The Visual Computer 23, 583 - 605.

Reeves, W., 1983. Particle systems - a technique for modeling a class of fuzzy objects. Computer Graphics 17 (3), 359-376. 
Reinhardt, E., Crookston, N. L., 2003. Fire and fuels extension to the forest vegetation simulator (ffe fvs). Tech. rep., Gen. Tech. Rep. RMRS-GTR116. Ogden, UT: U.S. Department of Agriculture, Forest Service, Rocky Mountain Research Station. 209 p.

Serón, F. J., Gutiérrez, D., Magallón, J., Ferragut, L., Asensio, M. I., 2005. The evolution of a wildland forest fire front. International Journal of Computer Graphics 21 (3), 152 - 169.

Sherman, W., Penick, M., Su, S., Brown, T., Harris, F., 2007a. VRFire: an Immersive Visualization Experience for Wildfire Spread Analysis. Proc. of IEEE VR 2007, March 12-15. Charlotte, NC.

Sherman, W. R., Su, S., McDonald, P. A., Mu, Y., Jr., F. H., March-April 2007b. Open-source tools for immersive environmental visualization. IEEE Computer Graphics and Applications 27 (2), 88-91.

Smith, S., Ericson, E., 2009. Using immersive game-based virtual reality to teach fire-safety skills to children. Virtual Reality 13, 8799.

Su, S., Sherman, W., Harris, F., Dye, M., 2006. Taverns: Visualization and manipulation of gis data in 3d large screen immersive environments. In: 16th International Conference on Artificial Reality and TelexistenceWorkshops (ICAT'06). pp. 656-661.

Suárez, J., Plaza, A., 2009. Four-triangles adaptive algorithms for rtin terrain meshes. Mathematical and Computer Modelling 49 (5-6), 1012 - 1020.

Sullivan, A., 2008. A review of wildland fire spread modelling, 1990-present. Department of Theoretical Physics, The Australian National University. 
405 Thon, S., Remy, E., Raffin, R., Gesquiére, G., 2007. Combining GIS and 406 forest fire simulation in a virtual reality environment for environmental 407 management. Architecture, City and Environment, 2:4.

408 Williams, B. J., Song, B., Hom, J., Duveneck, M., 2008. Wildfire visualization 409 using GIS and forest inventory data. In: Proceedings of the 6th Southern ${ }_{410} \quad$ Forestry and Natural Resources GIS Conference 2008. 